\title{
Successful remission of ulcerative colitis flare-up during pregnancy with adsorptive granulomonocytapheresis plus tacrolimus
}

\author{
Tomoyoshi Shibuya, Keiichi Haga, Masato Kamei, Koki Okahara, Shoko Ito, Masahito Takahashi, Osamu \\ Nomura, Takashi Murakami, Masae Makino, Tomohiro Kodani, Dai Ishikawa, Naoto Sakamoto, Taro \\ Osada, Tatsuo Ogihara, Sumio Watanabe, Akihito Nagahara \\ Department of Gastroenterology, Juntendo University School of Medicine, Tokyo, Japan
}

Ulcerative colitis (UC) is 1 of the 2 major phenotypes of chronic inflammatory bowel disease (IBD), which afflicts millions of individuals throughout the world with debilitating symptoms that impair function and quality of life. Further, IBD often affects women during childbearing age. Indeed, UC activity frequently increases during pregnancy, and the medications used to induce remission may adversely affect the health of the mother and the unborn child. We report successful induction of a remission in a UC case who experienced a flare-up in the first trimester of pregnancy. Upon relapse, she was treated with steroids and adsorptive granulomonocytapheresis (GMA) with the Adacolumn plus tacrolimus. This combination therapy induced a stable remission that was maintained during her entire pregnancy. She gave birth to a healthy child at 36 weeks of pregnancy with no maternal or fetal complications. Our experience indicates that GMA, as a non-drug therapeutic intervention with a favorable safety profile, plus tacrolimus might be a relevant treatment option for patients with active IBD during pregnancy. A future study of a large cohort of pregnant patients should strengthen our findings. (Intest Res 2018;16:484-488)

Key Words: Colitis, ulcerative; Pregnancy; Tacrolimus; Granulomonocytapheresis

\section{INTRODUCTION}

Ulcerative colitis (UC) is 1 of the 2 major phenotypes of chronic IBD, which afflicts millions of individuals throughout the world with debilitating symptoms that impair function and quality of life. Further, UC frequently affects women of childbearing age.

Tacrolimus is a calcineurin inhibitor, which inhibits inflammatory cytokine release by activated T-cells. Historically tacrolimus has been known as an immunosuppressant used orally and has shown efficacy in patients undergoing organ

Received November 21, 2017. Revised February 1, 2018

Accepted February 26, 2018. Published online April 6, 2018

Correspondence to Tomoyoshi Shibuya, Department of Gastroenterology,

Juntendo University School of Medicine, 2-1-1 Hongo, Bunkyo-ku, Tokyo

113-8421, Japan. Tel: +81-3-5802-1058, Fax: +81-3-3813-8862, E-mail:

tomoyosi@juntendo.ac.jp transplantation or affected by an autoimmune disease. ${ }^{1}$ Recently, tacrolimus has found applications in UC patients. ${ }^{2}$ With this background in mind, we thought that tacrolimus might be an effective medication for UC during pregnancy. However, to our knowledge, hitherto no study has evaluated the safety of tacrolimus in pregnant UC patients or on pregnancy outcome. Here we report a case that experienced a UC flare-up during pregnancy and was successfully treated with tacrolimus and granulomonocytapheresis (GMA) with the Adacolumn.

\section{CASE REPORT}

A 38-year-old woman with UC and in the 5th gestational week of pregnancy came to Juntendo Hospital to ask about the risks of UC and the use of medications in general in relation to her pregnancy outcome. Her medical history includ-

\footnotetext{
๑ Copyright 2018. Korean Association for the Study of Intestinal Diseases. All rights reserved.

This is an Open Access article distributed under the terms of the Creative Commons Attribution Non-Commercial License (http://creativecommons.org/licenses/by-nc/4.0)

which permits unrestricted non-commercial use, distribution, and reproduction in any medium, provided the original work is properly cited.
} 
ed a diagnosis of UC (relapse-remitting, total colitis type), which was made 13 years previously. Additionally, she had had serious allergic reactions to mesalazine. She had started treatment with tacrolimus at 4-6 $\mathrm{mg}$ /day and prednisolone sodium phosphate solution 3 years previous to this visit. At our hospital, we decided to discontinue tacrolimus administration because her UC clinical activity index (CAI) was 4 (close to remission level). After 1 week, she experienced a relapse with hematochezia and diarrhea $(\mathrm{CAI}=13)$. We ar-

Table 1. Blood Test and Fecal Culture Results on Admission

\begin{tabular}{|c|c|}
\hline List & Value \\
\hline WBC $\left(\times 10^{6} / L\right)$ & 29,500 \\
\hline $\mathrm{RBC}\left(\times 10^{6} / \mathrm{L}\right)$ & 369 \\
\hline Hemoglobin (g/dL) & 10.7 \\
\hline Hematocrit (\%) & 30.7 \\
\hline $\operatorname{Plt}\left(\times 10^{10} / L\right)$ & 53.8 \\
\hline $\mathrm{ESR}(\mathrm{mm} / \mathrm{hr})$ & 84 \\
\hline $\mathrm{CRP}(\mathrm{mg} / \mathrm{dL})$ & 12.0 \\
\hline $\operatorname{ALP}(U / L)$ & 262 \\
\hline AST (U/L) & 12 \\
\hline $\operatorname{ALT}(\mathrm{U} / \mathrm{L})$ & 7 \\
\hline $\mathrm{LD}(\mathrm{U} / \mathrm{L})$ & 144 \\
\hline TB (mg/dL) & 0.19 \\
\hline $\operatorname{ChE}(\mathrm{U} / \mathrm{L})$ & 165 \\
\hline $\mathrm{TP}(\mathrm{g} / \mathrm{dL})$ & 6.9 \\
\hline Albumin (g/dL) & 3.0 \\
\hline T-cho (mg/dL) & 125 \\
\hline Glu (mg/dL) & 97 \\
\hline BUN (mg/dL) & 37 \\
\hline Creatinine (mg/dL) & 2.53 \\
\hline eGFR $\left(\mathrm{mL} / \mathrm{min} / 1.73 \mathrm{~m}^{2}\right)$ & 18.3 \\
\hline $\mathrm{Na}(\mathrm{mmol} / \mathrm{L})$ & 134 \\
\hline $\mathrm{K}(\mathrm{mmol} / \mathrm{L})$ & 4.1 \\
\hline $\mathrm{Cl}(\mathrm{mmol} / \mathrm{L})$ & 95 \\
\hline C7-HRP & Negative \\
\hline \multicolumn{2}{|l|}{ Fecal culture } \\
\hline Common bacterium & Negative \\
\hline Acid-fast bacterium & Negative \\
\hline
\end{tabular}

WBC, white blood cell; RBC, red blood cell; PIt, platelets; LD, lactate dehydrogenase; $\mathrm{TB}$, total bilirubin; $\mathrm{ChE}$, cholinesterase; $\mathrm{TP}$, total protein; T-cho, total cholesterol; Glu, glucose; eGFR, estimated glomerular filtration rate; $\mathrm{C} 7-\mathrm{HRP}$, cytomegalovirus antigenemia. ranged a conference with an experienced obstetrician after which we re-administered tacrolimus at $6 \mathrm{mg} /$ day. However, due to hyperemesis gravidarum, she could not take any medicine orally and developed dehydration as well as an acute renal disorder. Therefore, tacrolimus was discontinued and she was hospitalized. As shown in the Table 1, the patient's serum creatinine level was $2.53 \mathrm{mg} / \mathrm{dL}$ on admission together with severe UC (CAI=16). In hospital, she was administered corticosteroid at $40 \mathrm{mg} / \mathrm{day}(1 \mathrm{mg} / \mathrm{kg}$, body weight) plus GMA therapy with the Adacolumn, but a full remission was not achieved after 3 weeks (CAI=6). Meanwhile, her renal function recovered following fluid replacement therapy (creatinine, $0.61 \mathrm{mg} / \mathrm{dL}$ ). Following another consultation with the obstetrician, we re-started tacrolimus at 3 $\mathrm{mg} /$ day, gradually increasing the dose to reach a high trough plasma concentration of $10-15 \mathrm{ng} / \mathrm{mL}$, and maintained this dosage for 2 weeks. She achieved a stable remission $(\mathrm{CAI}=4)$ and was discharged from hospital. She continued taking tacrolimus, achieving a low trough plasma level of $5-10 \mathrm{ng} / \mathrm{mL}$ in an outpatient setting (Fig. 1).

This patient continued oral tacrolimus throughout her pregnancy and was able to discontinue corticosteroid. She delivered electively at 36 weeks and 3 days by cesarean section a healthy baby boy (Apgar score 9/10) with a birth weight of 2,296 g. She was discharged from hospital at postoperative day 5 . The baby is now 10 months old and growing well. There is no evidence of any functional impairment or developmental delay.

\section{DISCUSSION}

This case with active UC during pregnancy and intolerant to first-line salicylate preparations demonstrates a serious challenge in therapeutic settings. When active IBD develops at the time of conception the disease activity as well as the medical therapy must be viewed as two independent factors that may adversely impact the pregnancy outcome. Indeed, higher relapse rates have been observed in pregnant IBD patients during the first 2 trimesters. This underscores the importance of advising patients to try to conceive during a stable remission. ${ }^{3,4}$

There are multiple reports on steroids, salicylates, thiopurines, and anti-tumor necrosis factor (anti-TNF) biologics in the treatment of patients with active UC during pregnancy, but there are inadequate reports on tacrolimus and GMA in this setting. For over 9 years, we have been using GMA as a non-drug therapeutic option to treat our regular IBD patients. GMA is effective, but its appropriate use might be 


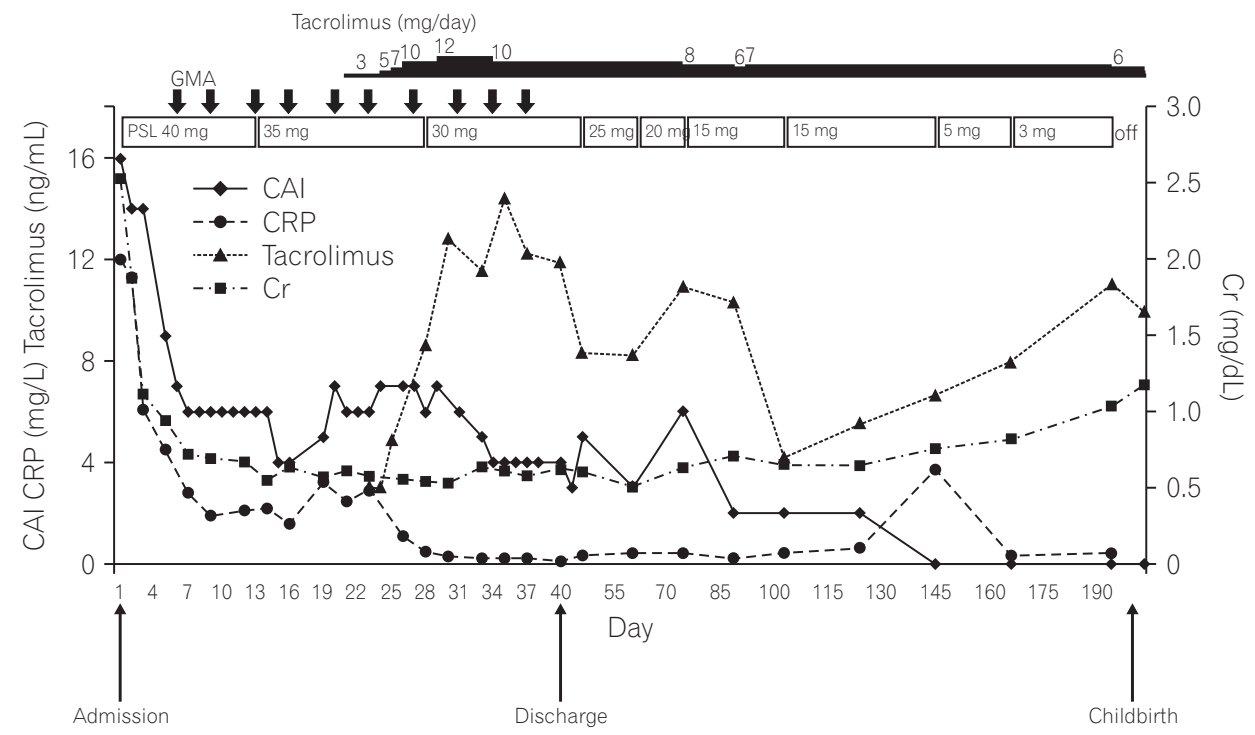

Fig. 1. Clinical course of a pregnant patient who achieved clinical remission during treatment with adsorptive granulomonocytapheresis (GMA) with the Adacolumn in combination with tacrolimus. The bold down arrows indicate GMA. CAl, clinical activity index; PSL, prednisolone; $\mathrm{Cr}$, creatinine. overlooked if started after patients are first exposed to multiple drugs and the disease has become severe and refractory to drugs. ${ }^{5,6}$ Other subgroups in which GMA has shown efficacy include those in whom immunomodulator drugs are ineffective or are not tolerated. GMA has had a good safety profile, and adverse side effects are uncommon and never severe. ${ }^{5-7}$ Nonetheless, to our knowledge, only a few reports are so far available in the literature about the safety and efficacy of GMA for active UC during pregnancy. Takahashi et $\mathrm{al}^{7}{ }^{7}$ used GMA to treat 3 patients who experienced relapse during pregnancy. The number of GMA treatment sessions applied was 5 to 10. All 3 patients achieved remission, 2 with mucosal healing as well. They reported that GMA as a nonpharmacologic treatment was effective and safe. Although 1 of the 3 cases did not respond well to the initial GMA treatment sessions, a moderate dose of prednisolone enhanced the efficacy of GMA, and tapering of the prednisolone dose shortly after remission was not associated with relapse. ${ }^{7}$ Also our patient responded to GMA, but did not achieve full remission. Because the patient was pregnant, we avoided colonoscopy to assess her UC severity. However, at the time of confirmation of her pregnancy, the patient was in clinical remission $(\mathrm{CAI}=4)$. Therefore, we discontinued tacrolimus administration, but soon after the discontinuation she experienced a flare-up. From her clinical records, we were aware that tacrolimus was effective for inducing remission in this patient. Other likely options would have been anti-TNF- $\alpha$ if she had not responded to tacrolimus.

Like cyclosporine, tacrolimus is widely used for the suppression of graft versus host reactions after bone marrow transplantation or to inhibit rejection after solid organ transplantation. Therefore, hitherto data on pregnancy outcome associated with the use of tacrolimus have mainly relied on non-IBD patients. In women who had undergone kidney transplantation, in the pregnancy guidelines, it is recommended to continue immunosuppressive therapy by cyclosporine or tacrolimus. ${ }^{8}$ However, despite this recommendation, ${ }^{8}$ in IBD patients, evidence for cyclosporine is limited to a small number of women who experienced severe relapses of UC during pregnancy. ${ }^{9-11}$ Only a single case report on tacrolimus in a pregnant UC patient has been published. ${ }^{12}$ No obvious congenital malformations were described in infants of pregnant patients who were treated by either cyclosporine or tacrolimus, although some outcomes were complicated by prematurity and low birth weight. However, it is difficult to differentiate the impact of severe disease from an adverse effect of a drug.

In nonpregnant UC patients, tacrolimus was associated with high clinical response without an increased risk of severe adverse events. ${ }^{13}$ Accordingly, several guidelines now recommend using tacrolimus for steroid-refractory active UC. ${ }^{14,15}$ However, currently data on the effect of tacrolimus in pregnant patients and pregnancy outcomes are inadequate. Therefore, physicians may advise women to avoid conception during tacrolimus therapy. Further, although tacrolimus readily crosses the maternal-fetal blood barrier, ${ }^{16}$ there have been reports of many successful pregnancies in those who have had solid organ transplantations with the incidence of congenital abnormalities not higher than in the general population. ${ }^{17,18}$ In one study, of 100 pregnancies in 84 
women treated with tacrolimus, 4 infants had abnormalities, but there was no consistent pattern of anatomical malformation. ${ }^{19}$ In our UC patient, tacrolimus induced a stable remission with no obvious maternal or fetal complications. Finally, the safety of tacrolimus has not been determined, but its effectiveness in treating active UC indicates that its benefits are greater than potential risks that have been suspected but not confirmed.

In conclusion, our experience in this case suggests that tacrolimus in combination with adsorptive GMA may be a safe and effective treatment option for patients with UC who experience flare-ups during pregnancy. In particular, patients whether or not pregnant who are intolerant to conventional medications could try tacrolimus. However, we strongly believe that future trials with large cohorts of pregnant patients are warranted to determine the optimal regimen and longterm maternal and fetal safety associated with tacrolimus therapy.

\section{FINANCIAL SUPPORT}

The authors received no financial support for the research, authorship, and/or publication of this article.

\section{CONFLICT OF INTEREST}

No potential conflict of interest relevant to this article was reported.

\section{AUTHOR CONTRIBUTION}

T.S., K.H. wrote the case report. M.K. described the figure. All authors were involved in critical revision of the manuscript and have approved the final manuscript for submission.

\section{REFERENCES}

1. Barbarino JM, Staatz CE, Venkataramanan R, Klein TE, Altman RB. PharmGKB summary: cyclosporine and tacrolimus pathways. Pharmacogenet Genomics 2013;23:563-585.

2. Harbord M, Eliakim R, Bettenworth D, et al. Third European evidence-based consensus on diagnosis and management of ulcerative colitis. Part 2: current management. J Crohns Colitis 2017;11:769-784.
3. Mahadevan U, Sandborn WJ, Li DK, Hakimian S, Kane S, Corley DA. Pregnancy outcomes in women with inflammatory bowel disease: a large community-based study from Northern California. Gastroenterology 2007;133:1106-1112.

4. Pedersen N, Bortoli A, Duricova D, et al. The course of inflammatory bowel disease during pregnancy and postpartum: a prospective European ECCO-EpiCom Study of 209 pregnant women. Aliment Pharmacol Ther 2013;38:501-512.

5. Hibi T, Sameshima Y, Sekiguchi Y, et al. Treating ulcerative colitis by Adacolumn therapeutic leucocytapheresis: clinical efficacy and safety based on surveillance of 656 patients in 53 centres in Japan. Dig Liver Dis 2009;41:570-577.

6. Saniabadi AR, Hanai H, Suzuki Y, et al. Adacolumn for selective leukocytapheresis as a non-pharmacological treatment for patients with disorders of the immune system: an adjunct or an alternative to drug therapy? J Clin Apher 2005;20:171-184.

7. Takahashi H, Sugawara K, Sugimura M, et al. Flare up of ulcerative colitis during pregnancy treated by adsorptive granulocyte and monocyte apheresis: therapeutic outcomes in three pregnant patients. Arch Gynecol Obstet 2013;288:341-347.

8. EBPG Expert Group on Renal Transplantation. European best practice guidelines for renal transplantation. Section IV: Longterm management of the transplant recipient. IV.10. Pregnancy in renal transplant recipients. Nephrol Dial Transplant 2002;17 Suppl 4:50-55.

9. Korelitz BI. Inflammatory bowel disease and pregnancy. Gastroenterol Clin North Am 1998;27:213-224.

10. Hviid A, Mølgaard-Nielsen D. Corticosteroid use during pregnancy and risk of orofacial clefts. CMAJ 2011;183:796-804.

11. Branche J, Cortot A, Bourreille A, et al. Cyclosporine treatment of steroid-refractory ulcerative colitis during pregnancy. Inflamm Bowel Dis 2009;15:1044-1048.

12. Baumgart DC, Sturm A, Wiedenmann B, Dignass AU. Uneventful pregnancy and neonatal outcome with tacrolimus in refractory ulcerative colitis. Gut 2005;54:1822-1823.

13. Ogata H, Kato J, Hirai F, et al. Double-blind, placebo-controlled trial of oral tacrolimus (FK506) in the management of hospitalized patients with steroid-refractory ulcerative colitis. Inflamm Bowel Dis 2012;18:803-808.

14. Marri SR, Ahn C, Buchman AL. Voluntary childlessness is increased in women with inflammatory bowel disease. Inflamm Bowel Dis 2007;13:591-599.

15. Tavernier N, Fumery M, Peyrin-Biroulet L, Colombel JF, GowerRousseau C. Systematic review: fertility in non-surgically treated inflammatory bowel disease. Aliment Pharmacol Ther 2013;38:847-853. 
16. Pérgola PE, Kancharla A, Riley DJ. Kidney transplantation during the first trimester of pregnancy: immunosuppression with mycophenolate mofetil, tacrolimus, and prednisone. Transplantation 2001;71:994-997.

17. Armenti VT, Constantinescu S, Moritz MJ, Davison JM. Pregnancy after transplantation. Transplant Rev (Orlando) 2008;22:223-240.
18. Khan SS, Constantinescu S, Silva P, Coscia LA, Armenti VT. Pregnancy outcomes in renal transplant recipients on Neoral vs. tacrolimus. J Am Soc Nephrol 2006;17:562A.

19. Kainz A, Harabacz I, Cowlrick IS, Gadgil SD, Hagiwara D. Review of the course and outcome of 100 pregnancies in 84 women treated with tacrolimus. Transplantation 2000;70:17181721 . 